\title{
A Novel Thermal Swelling Model for a Rechargeable Lithium-ion Battery Cell
}

\author{
Ki-Yong Oh ${ }^{\mathrm{a}}$, and Bogdan I. Epureanu ${ }^{\mathrm{a}, *}$ \\ ${ }^{\mathrm{a}}$ Department of Mechanical Engineering, University of Michigan, 2350 Hayward Street, Ann
}

Arbor, MI 48109-2125,USA

*Corresponding author: Tel.: +1-734-647-6391; fax: +1-734-615-6647

E-mail address: epureanu@umich.edu

\begin{abstract}
The thermal swelling of rechargeable lithium-ion battery cells is investigated as a function of the charge state and the charge/discharge rate. The thermal swelling shows significant dependency on the state of charge and the charge rate. The thermal swelling follows a quadratic form at low temperatures, and shows linear characteristics with respect to temperature at high temperatures in free-swelling conditions. Moreover, the equivalent coefficient of thermal expansion is much larger than that of each electrode and host materials, suggesting that the separator and the complex shape of the cell play a critical role in thermal expansion. Based on the experimental characterization, a novel thermal swelling model is proposed. The model introduces an equivalent coefficient of thermal expansion for the cell and also considers the temperature distribution throughout the battery by using heat transfer theory. The comparison between the proposed model and experiments demonstrates that the model accurately predicts thermal swelling at a variety of charge/discharge rates during operation and relaxation periods. The
\end{abstract}


model is relatively simple yet very accurate. Hence, it can be useful for battery management applied to prolong the cycle life of cells and packs.

\section{Keywords}

Lithium-ion battery; phase transition; swelling; thermal expansion; the coefficient of thermal expansion

\section{Introduction}

Concerns for energy security, instability in world oil markets, and limitations of carbon emissions have accelerated the development of eco-friendly, high-efficiency automobiles. This drives automobile industries toward the development of vehicle electrification technology. Electrified vehicles currently use lithium-ion (Li-ion) batteries as the reversible power source. Li-ion batteries have advantages such as high power/energy density, high potential, and low selfdischarge rate. They are also environmentally friendly and have a long life cycle [1-3].

While vehicle electrification with the advent of the Li-ion batteries [4] enhances fuel efficiency and reduces $\mathrm{CO}_{2}$ emissions, many challenges still exist when using Li-ion batteries such as their limited performance at low temperatures [5] and their thermal runaway [6]. Especially, extensive research on vehicle electrification has been driven by stringent safety standards for air and ground applications. Therefore, recent research focuses on the thermal distribution and the heat dissipation of Li-ion battery packs $[7,8]$ as elevated temperatures not only can cause thermal runaway but can also degrade battery life. A variety of heat transfer models have been created 
for the Li-ion batteries and validated through experiments [9-12]. Many methods and strategies for use in battery management systems (BMS) have been developed to mitigate the safety concerns while enhancing efficiency and capabilities [13,14,24].

Heat is generated in a Li-ion battery cell from two sources: entropy change and Joule heat. Entropy compensates the residual energy in the energy conversion process between the enthalpy and the Gibbs free energy [15]. Entropy heat is therefore reversible; it is generally endothermic during charge and exothermic during discharge. Joule heat is due to the internal resistance of the cell components such as the positive/negative electrodes and the separator. Joule heat is irreversible and exothermic regardless of the charge process. These sources of heat not only change the temperature but also change the volume of the Li-ion battery. This volume change causes additional periodic thermal stress during operation and affects the lifespan of the cells and packs. Therefore, many efforts have been devoted to characterize the thermal expansion of the host materials in micro-scale [16-18]. These studies provide a useful foundation for understanding the thermal characteristics of the active materials. However, the dynamic thermal mechanics at the cell-level are more complicated because of the hundreds of contact surfaces between electrodes and mechanical constraints such as wounding shape of the jellyroll and clamping current collectors with bus bars in the edge sides. Our previous study showed for the first time that thermal swelling is similar in order of magnitude with Li-ion intercalation swelling and thereby far from insignificant [19]. However, studies of thermal expansion on the cell-level are still few, making it difficult to estimate the thermal stress due to thermal expansion, while the swelling due to electrochemical reaction, i.e. Li-ion intercalation, has intensively been investigated from micro-scale to macro-scale [20-23]. Especially, the most important property which comes from both the material and its structure inside the cell, i.e. the equivalent 
coefficient of thermal expansion on the cell-level, was never reported before, even though this property is crucial not only to predict the dynamic thermal mechanics of battery cells but also to estimate periodic thermal stress/strain on packs. Moreover, the modeling of the thermal expansion on the cell-level with the measured material property has not been investigated in great detail, although characterizing the thermal expansion has become important to prolong the life of Li-ion batteries. The limited information about thermal stress and strain on the cell-level stimulates the quantification of thermal stress and strain on the battery cells and packs, which affect the battery performance in packs.

In this paper, we report results and models obtained by measuring the expansion of a cell. Highprecision displacement sensors were used to measure the cell-level swelling arising from charge/discharge of an unconstrained graphite/nickel-manganese-cobalt-oxide (NMC) cell whose temperature was regulated in a thermal chamber. The measured thermal expansion was observed to vary significantly with experimental control parameters and also was observed to exhibit nonlinear characteristics with respect to temperature. An equivalent coefficient of the thermal expansion was calculated as a function of the state-of-charge (SOC) at a variety of locations. A novel thermal swelling is proposed based on the experimental results. The proposed model uses the measured equivalent coefficient of thermal expansion to estimate the thermal swelling. Moreover, a 1-D heat conduction model is introduced to account for the temperature distribution through the cell for a more accurate estimation of thermal swelling. The proposed model was verified by comparisons with experimental data consisting of thermal expansion at various C-rates, both during operation and relaxation periods. The experimental validations confirm that the proposed model accurately predicts experimental observations in a variety of 
operational conditions. Such an accurately model, able to estimate cell thermal behavior, may be beneficial to the design and management of not only single cells but also battery packs.

\section{Experiments}

This study used a flat-wound type prismatic 5Ah Li-ion cell obtained from a Ford Fusion HEV battery pack. Detailed information of the Li-ion cell is available in Ref. [19].

The free swelling of the cell was measured with high-precision contact-type displacement sensors with $1 \mu \mathrm{m}$ accuracy and $0.1 \mu \mathrm{m}$ resolution (Keyence GT2-H12KL, Japan). The sensor head creates a contact force on the battery surface of less than $0.3 \mathrm{~N}$. The sensor was installed in a fixture as shown in Figure 1. The fixture was made from ABS plastic using a rapid-prototyping machine (Dimension Elite FDM, USA). The prismatic cell was constrained at its eight corners with ABS plastic set-screws in the fixture, but was otherwise unconstrained. The fixture was placed inside a thermal chamber (ESPEC BTZ-133, Japan) with controlled desired temperature. Two thermocouples were also used. One thermocouple was installed on the center of the cell to measure cell surface temperature (in a location that avoided interference with the displacement sensors). The other thermocouple was installed between the fixture and the cell to measure nearsurface ambient temperature.

A first experiment was carried out to assess the thermal expansion characteristics of the cell over the SOC. In this experiment, relative thermal expansion in the $z$ direction, which is perpendicular to the multi-layer electrode sandwich, was measured with a variety of SOCs at five locations labeled 1-5 in Figure 1. Prior to discharge, the battery was fully charged using a 
standard constant current, constant voltage charging profile at $2 \mathrm{~A}(0.4 \mathrm{C})$ at $25^{\circ} \mathrm{C}$; the voltage was clamped after reaching $4.1 \mathrm{~V}$, at which it was held until the current tapered to $\mathrm{C} / 100(50 \mathrm{~mA})$. To obtain the desired SOC, the battery was discharged with a $0.4 \mathrm{C}$ current for an appropriate time. For example, 1.25 hour discharge time was used to obtain a 50\% SOC. The battery was allowed to rest at open circuit for 1 hour to ensure full relaxation at the end of discharge. Then, the temperature of the thermal chamber was incrementally changed from $5^{\circ} \mathrm{C}$ to $45^{\circ} \mathrm{C}$ with $5^{\circ} \mathrm{C}$ increments. Each temperature variation was followed by 5 hours of rest time to ensure thermal equilibrium. This procedure was repeated from $0 \%$ to $100 \%$ SOC with $25 \%$ SOC increments.

The second experiment repeated the same temperature profile (from $5^{\circ} \mathrm{C}$ to $45^{\circ} \mathrm{C}$ with a $5^{\circ} \mathrm{C}$ increment) of the first experiment with an aluminum block instead of the cell. That was done to characterize the thermal expansion of the fixture and the sensors because they also expand or shrink when the ambient temperature varies. Five sensors were installed on one side of the cell at the same locations as in the first experiment. One other sensor was installed in the center of the opposite face of the cell (without contacting the cell) to observe the thermal behavior of the sensor itself.

In a third experiment, swelling at several C-rates was measured for the validation of the thermal swelling model. The net displacement at the center of the battery was measured with respect to the charge state during discharge using a standard constant current discharging profile. All discharges were performed down to 3.0V. Measurements with exterior thermocouples showed that the lowest current used, 0.2C (1A), did not cause significant heating. The battery-cell surface remained within $0.1^{\circ} \mathrm{C}$ of the $25^{\circ} \mathrm{C}$ ambient temperature during discharge. Data at $0.2 \mathrm{C}$ therefore allows direct correlations to be made between swelling and Li-ion intercalation in a cell sandwich without significant convolution by thermal expansion. In contrast, the cell surface 
temperature deviated more significantly from the ambient at high C-rate tests where temperature variations produced considerable thermal swelling.

In a fourth and last experiment, pulse excitation experiment was carried out to assess the viability of the proposed model in HEVs. The displacement sensors were placed at the geometric centers on both faces of the cell normal to the cell surface (location 3 shown in Figure 1). The battery was fully charged using standard CCCV protocol prior to discharge. Then, the battery was discharged with a $2 \mathrm{~A}(0.4 \mathrm{C})$ current for 1.25 hour to obtain a $50 \%$ SOC. The battery was allowed to rest at open circuit for 3 hours at the end of charge and discharge respectively. Then, pulse current was induced to the battery cell at 7.8 C (39A) with twenty-second (10 second charge and 10 second discharge) period for 720 seconds to obtain pure thermal expansion. The induced current was the maximum current in the current setup. The maximum capability of the battery test equipment is $40 \mathrm{~A}$. The SOC is minimally changed during pulse excitation, suggesting that the swelling in this experiment originates from the resistive heating, and thereby the swelling measured can be considered as pure thermal swelling.

\section{Thermal swelling model}

When a jellyroll is fabricated, positive/separator/negative electrodes are wound. The individual layered sheets are thin. The thickness of each component is of the order of dozens of micrometers. Hence, lumped parameters - which represent equivalent material properties of stacks of thin individual anode/separator/cathode layers - are frequently used to describe the 
thermal behavior of the cell [24,25]. Moreover, it is reasonable to assume uniform heat generation through the cell [26-28].

Prior to operation (charge or discharge), the temperature is constant through the cell and the same as the ambient temperature because the cell is at thermal equilibrium. However, temperature variations occur during operation through the cell. Assuming uniform heat generation and lumped parameters, the temperature distribution across the cell thickness is parabolic, as shown in Figure 2 [28]. This temperature distribution is due to the assumed uniform heating caused by Joule heat and entropy heat. The non-uniform temperature distribution is important when estimating the thermal swelling of the cell. The thermal swelling normal to $z$ direction (shown in Figure 1) can be expressed as

$$
s_{h}=\alpha \int_{0}^{L}\left(T(x)-T_{S_{i}}\right) d x
$$

where $s_{h}, L, \alpha, T$ represent the thermal swelling (due to cell heating/cooling), the original thickness of the cell, the (lumped) coefficient of thermal expansion, and the temperature, while subscript $S_{i}$ denotes the initial state of the surface.

Similar to the modulus of elasticity and the interlayer spacing of the graphite lattice $[29,30]$, the equivalent coefficient of thermal expansion $\alpha$ can be affected by the phase transition of the electrodes. Thus, $\alpha$ is a function of SOC, which varies in time. Hence, $\alpha$ varies in time.

A linear temperature distribution across the cell is obtained for a uniform heat generation. The corresponding thermal swelling can be computed by integrating Eq. (1) to obtain 


$$
s_{h}(t)=\alpha(t) L\left[\frac{2}{3}\left(T_{C}(t)-T_{S}(t)\right)-\left(T_{S}(t)-T_{R e f}\right)\right]
$$

where $t$ is time, $T_{R e f}$ is a reference (ambient) temperature, while $T_{C}$ and $T_{S}$ are the core and surface temperatures which vary in time. The reference temperature $T_{R e f}$ is equal to the surface temperature $T_{S_{i}}$ at the initial state because the cell is initially at thermal equilibrium.

The first term, $\frac{2}{3} \alpha(t) L\left(T_{C}(t)-T_{S}(t)\right)$, is a component of the thermal swelling due to temperature variation across the cell. The second term, $\alpha(t) L\left(T_{S}(t)-T_{\text {Ref }}\right)$, is the component of the thermal swelling due to the difference between the current surface temperature and the reference temperature.

The core, surface, and ambient temperatures, and the equivalent coefficient of thermal expansion are needed to estimate the thermal swelling in Eq. (2). The ambient and surface temperatures, and the equivalent coefficient of thermal expansion of the cell, are measureable and thereby available. However, it is hard to measure the core temperature of the cell. Thus, to predict the core temperature during operation, a 1-D heat conduction model is used. This model can be expressed as

$$
C_{\text {cell }} \frac{d T_{C}(t)}{d t}=i^{2}(t) R+i(t) T_{C}(t) \frac{d U}{d T}(t)+2 K_{\text {cross }} \frac{A_{\text {cross }}}{L / 2}\left(T_{S}(t)-T_{C}(t)\right),
$$

where $C_{\text {cell }}, i, R, U, K_{\text {cross }}$ denote the cell heat capacity, the current, the electric resistivity, the potential, and the thermal conductivity for the through-plane direction (Figure 1) of the cell, while $A_{\text {cross }}$ denotes the cell surface area. This model includes two heat sources: Joule heat, $i^{2}(t) R$, and entropy heat, $i(t) T_{C}(t) \frac{d U}{d T}(t)$. 
During operation, the SOC is variable and hence the coefficient of thermal expansion is variable. In contrast, the SOC is constant during the relaxation period after a discharge, and no phase transitions occur. Hence, the coefficient of thermal expansion is constant during the relaxation period. The swelling and the core temperature during the relaxation period can be expressed as

$$
\begin{gathered}
s_{h}(t)=\alpha_{r e l} L\left[\frac{2}{3}\left(T_{C}(t)-T_{S}(t)\right)-\left(T_{S}(t)-T_{R e f}\right)\right], \\
C_{\text {cell }} \frac{d T_{C}(t)}{d t}=2 K_{\text {cross }} \frac{A_{\text {cross }}}{L / 2}\left(T_{S}(t)-T_{C}(t)\right),
\end{gathered}
$$

Therefore, the equivalent coefficient of thermal expansion for the relaxation period $\alpha_{r e l}$ can be obtained from Eq. (2) applied at the end of discharge. Also, Eq. (3) in the relaxation period can be written as Eq. (5). This shows that the core temperature during the relaxation period has a first order response similar to the surface temperature [31] as there is no heat source in the cell.

\section{Results and Discussion}

\subsection{Thermal behavior of the cell}

In a first experiment, a quasi-equilibrium thermal swelling of the cell was measured, ranging from $5^{\circ} \mathrm{C}$ to $45^{\circ} \mathrm{C}$ with $5^{\circ} \mathrm{C}$ increments. The measured thermal swelling has to be calibrated because the measured value is the total thermal expansion, which includes the expansion of the sensor and that of the fixture, as shown in Eq. (6). 


$$
s_{\text {measured }}=-s_{\text {fixture }}+s_{\text {sensor }}+s_{h},
$$

where $s_{\text {measured }}$ is the measured swelling, $s_{\text {fixture }}$ is the fixture swelling, and $s_{\text {sensor }}$ is the sensor swelling. The negative sign of $s_{\text {fixture }}$ captures the fact that the fixture holds the sensor used to measure swelling, and hence the sensor indicates a lower value when the fixture expands.

In the second experiment, the cell was replaced by an aluminum block of the same dimensions. Measuring the swelling in this experiment, and knowing the actual swelling of the aluminum block (by knowing the dimensions and the thermal expansion coefficient of aluminum) allowed us to identify the swelling of the sensor and the fixture $\left(-s_{\text {fixture }}+s_{\text {sensor }}\right)$. Also, the thermal swelling of the sensor $s_{\text {sensor }}$ was characterized by using the sensor installed on the opposite face of the cell without contacting the cell. Note that the exact thermal behavior of the sensor is not needed for the calibration. The combined swelling of the sensor and the fixture $\left(-s_{\text {fixture }}+s_{\text {sensor }}\right)$ was measured in the second experiment and that is enough to estimate the pure thermal swelling of the cell in Eq. (6). However, the thermal behavior of the sensors was evaluated to clearly quantify the root cause of the thermal expansion in the measured data.

Figure 3 (a) and (b) shows the thermal swelling of the sensor and the fixture. The symbols show measured values and the solid lines represent least-square fitted lines from the measured values. The thickness at $5^{\circ} \mathrm{C}$ is taken as reference. Also, the sensor output at $5^{\circ} \mathrm{C}$ is set to zero (the sensor measures relative displacements). The fitted lines are in excellent agreement with measured thermal expansion in that the coefficient of determination $\left(\mathrm{R}^{2}\right)$ is over 0.998 for all fitted data. 
The swelling of the sensor and the fixture is linear with the temperature (in the measured range). However, the thermal expansion of the fixture is slightly different at each measurement location. This difference might be due to the manufacturing process and the geometric complexity of the fixture. The fixture consists of five components (Figure 1) manufactured using a rapid prototyping machine. This manufacturing process is known to create significant internal residual stresses. Moreover, the fixture components are stressed also during assembling. This internal and assembly stresses are small. However, the measured swelling is of the order of tens of microns. Therefore, the effects of the internal stresses in the fixture may not be negligible. Moreover, steel bolts were used to assemble the fixture. They have a different coefficient of thermal expansion than the rest of the fixture which is made of ABS plastic. Steel has much smaller value for the coefficient of thermal expansion than ABS plastic. Thus, the fixture likely exhibits a nonisotropic thermal expansion. In conclusion, the measured thermal expansion should be calibrated point by point to estimate reliable, pure thermal expansion of the cell.

Figure 3 (c)-(g) illustrate the thermal swelling of the cell ranging from $5^{\circ} \mathrm{C}$ to $45^{\circ} \mathrm{C}$ at five locations on the cell. These thermal swelling values were calibrated by using Eq.(6). The symbols indicate calibrated thermal swelling of the cell, and the solid lines represent least-square fitted lines from measured data ranging from $25^{\circ} \mathrm{C}$ to $45^{\circ} \mathrm{C}$ in Figure 3 (c)-(g). The thermal swelling increases in a nonlinear fashion below $25^{\circ} \mathrm{C}$, and in a linear fashion above $25^{\circ} \mathrm{C}$ at all locations, regardless of SOC. This result suggests that the jellyroll initially has small gaps inside (e.g., between electrode layers [32]). When the jellyroll starts to swell in the low temperature range below $25^{\circ} \mathrm{C}$, these gaps are eliminated/filled first. Once the gaps are filled in the high temperature range above $25^{\circ} \mathrm{C}$, the thermal expansion increases linearly with the temperature 
because the gaps are already filled, and the thermal expansion of the jellyroll fully contributes the expansion of the cell casing.

The thermal swelling at point 3 in Figure 1 (i.e., the center of the battery) is the largest. The swelling decreases near the edges because of the mechanical constraints imposed by the battery casing. The stiffness of the casing near the edges is larger than in the center. Therefore, the center region is mechanically easier to dilate.

We also note a slight asymmetry in the shape of the thermal swelling, which translates in the asymmetry in the equivalent coefficient of thermal expansion shown in Figure $3(\mathrm{~h})$. That is similar to Li-ion intercalation swelling, and is due to the non-symmetric geometry of the position of the jellyroll inside the casing $[19,35]$. Note again that the measured thermal swelling is not the pure thermal swelling but the equivalent thermal swelling of the battery cell, which reflects mechanical constraints and the hundreds of contact surfaces between folds of the electrodes. Hence, mechanical constraints play a critical role in the thermal swelling of this system together with the thermal expansion of the jellyroll. Nonetheless, the shape (spatial distribution) of the thermal swelling is approximately parabolic based on measurements at five locations. The shape of the thermal swelling appears to be different from that of Li-ion intercalation swelling. That is not surprising as the thermal swelling has a different physical origin compared Li-ion intercalation swelling.

The values of the equivalent coefficient of thermal expansion can be calculated for the measured locations from the data collected from $25^{\circ} \mathrm{C}$ to $45^{\circ} \mathrm{C}$. The basic premise is that the thermal swelling varies linearly with the temperature in the range of operational temperatures of the cell, but it contributes to fill the empty space in the low temperature regions. 
The measured equivalent coefficient of thermal expansion shows a dependency also on SOC (Figure $3(\mathrm{~h})$ ). This dependency originates from the phase transition of both the graphite and nickel-manganese-cobalt-oxide electrodes under charge or discharge $[36,37]$ because the phase transition also accompanies the changes of material properties [38,39]. However, the maximum difference is within $10 \%$. This is not significant considering that a large amount of Li-ions move from a cathode to an anode during discharge.

Interestingly, the order of magnitude for the equivalent coefficient of thermal expansion of the cell is similar to that of a polyethylene and a polypropylene [34] (main materials for a separator [33]), while the coefficient of thermal expansion of host materials and electrodes is much smaller than that for the cell [16-18]. The coefficient of thermal expansion for a polyethylene is between $10^{-4}$ and $3 \cdot 10^{-3} \mathrm{~K}^{-1}$, and the coefficient of thermal expansion for polypropylene is $6.5 \cdot 10^{-5}$ to $0.9 \cdot 10^{-3} \mathrm{~K}^{-1}[40]$. Thus, the separator might play an important role in the thermal expansion. Note that the material properties of polymer materials significantly depend on many things such as the atomic structure, the density of materials, and the manufacturing process. Therefore, there is typically a wide range of variation in polymers, and that is a reason why much research has been carried out to measure the material properties of individual polymer materials [41].

$$
\alpha L_{\text {total }}=\sum \alpha_{i} L_{i}
$$

Specifically, a separator, current collector, cathode, and anode take $28 \%, 24 \%, 24 \%$, and $24 \%$ of the total thickness of the cell [19]. Therefore, the estimated coefficient of thermal expansion for a separator by using Eq. (7) varies from $2.5 \cdot 10^{-3} \mathrm{~K}^{-1}$ to $4.2 \cdot 10^{-3} \mathrm{~K}^{-1}$ upon location considering the stacked materials; the detail configuration of the jellyroll used is described in Ref. [42] where the cross-section view of the jellyroll and the geometry inside of the cell is available from X-ray 
tomography in Ref. [43] (not shown here for the sake of brevity). The coefficients of thermal expansion for the current collectors and the graphite anode are assumed to be $2.3 \cdot 10^{-5} \mathrm{~K}^{-1}$ and $5.5 \cdot 10^{-5} \mathrm{~K}^{-1}[16]$. The coefficient of thermal expansion for the NMC cathode is assumed to be the same as that for graphite (because the coefficient of thermal expansion for the NMC cathode is not available). Moreover, the coefficient of thermal expansion for an insulation sheet between the case and the jellyroll [42] is estimated to be $1.0 \cdot 10^{-3} \mathrm{~K}^{-1}$. Indeed there is a lack of information for this material; the only information available is that this insulation sheet is made from a polymer material. Note that the estimated coefficient of thermal expansion for a separator is a rough estimation because of the limited information available. The manufacturer did not provide detailed information due to confidentiality and intellectual property rights. Moreover, the thermal expansion of the cell also could be affected by the constraints from the casing and the mechanical magnification of ruffling $[32,44]$. In particular, the ruffling phenomenon is more severe in the wound cell than the stacked cell because of constrained conditions. Specifically, two winding edges of the jellyroll (top and bottom sides for this prismatic cell) are fixed by the packing foil and two current collectors (left and right sides for this prismatic cell) are clamped by bus bars. At these edges, the interface among cathode, separator, and anode films cannot move, while the rest of the jellyroll (away from the constrained edges) easily expands and bends with a periodic wavy-like profile when temperature is elevated. This bucking behavior is the most severe and thereby results in the formation of several gaps/voids between electrode films in the center region [44], especially for free-swelling conditions. This phenomenon is not unlike the behavior of a soft beam fixed at both sides and exposed to temperature variations. The beam can bend and buckle due to thermal expansion and constrained boundary conditions. The most 
significant bending occurs in the middle of the beam. Similar to that, the thermal expansion of a cell is the largest in the center of the cell and the shape of the thermal swelling is convex.

The thermal expansion of electrolytes may not affect the expansion of the case on the cell level due to several reasons. First, the thermal expansion of electrolyte is of a similar order of magnitude with that of current collectors or active materials $[45,46]$. That suggests that the increase of the porous inside the separator may be larger than that of electrolytes and thereby the expansion of electrolytes do not contribute to the expansion on the cell level. Second, the modulus of elasticity for an electrolyte is at least hundreds of times smaller than that for other materials - the estimate for the electrolyte is $1 \mathrm{MPa}$ [47] - suggesting that the thermal expansion of electrolytes minimally generate a reaction force on the contact surfaces, which affects the volume of the overall battery cell. Finally, the battery cell contains a lot of empty space [43]. Therefore, the increased volume of electrolytes due to the temperature elevation just fills the empty space inside of the cell considering its soft modulus of elasticity.

In summary, both separators and mechanical constraints might contribute to the thermal expansion of the cell, even though it is hard to estimate the exact amount of contribution caused by each.

\subsection{Estimation of core temperature and thermal swelling}

The proposed thermal swelling model needs the core temperature of the cell. Therefore, a 1-D heat conduction model is introduced to predict the core temperature. The 1-D heat conduction model needs the equivalent thermal properties of the cell. The other side of this relationship is 
that the equivalent thermal properties of the cell can be characterized with the core and surface temperatures. Moreover, the core temperature can be calculated from the thermal swelling model, Eq. (2), if the (pure) thermal swelling of the cell is available and measured. In this paper, the equivalent thermal properties of the cell were parameterized with the predicted core temperature, which is estimated from the 5.0C thermal swelling data during discharge. Then, for validation, the 1-D heat conduction model with these thermal properties is applied to predict the thermal swelling at a variety of C-rates during operation as well as during relaxation periods. Details of the validation are described in the next subsection.

When the coefficient of thermal expansion is known, the core temperature can be calculated using measured quantities, namely: thermal swelling of the cell, surface temperature, and ambient/reference temperature. Eq. (2) can be rearranged to obtain the core temperature as

$$
T_{C}=\frac{3}{2}\left[\frac{s_{h}}{\alpha L}-\frac{T_{S}}{3}+T_{R e f}\right],
$$

However, this equation applies to pure thermal swelling $s_{h}$ (i.e. no Li-ion intercalation swelling). That is hard to obtain experimentally during charge or discharge directly because Liion intercalation swelling and thermal swelling occur simultaneously during operation. Instead of attempting to measure pure thermal swelling, we subtract the Li-ion swelling from the total swelling. This calculation has two premises. First, the total swelling is a sum of the Li-ion intercalation swelling and the thermal swelling. Second, the dependency of the Li-ion intercalation swelling on the C-rate is negligible. For such cases one obtains

$$
s_{h}=s_{T}-s_{L i},
$$


where $s_{h}, s_{T}, s_{L i}$ denote the thermal swelling, total swelling, and Li-ion intercalation swelling. The measured swelling at $0.2 \mathrm{C}$ (a dotted line in the top of Figure 4) is assumed to be pure Li-ion intercalation swelling $s_{L i}$ because the temperature variations during discharge at $0.2 \mathrm{C}$ are below $0.1^{\circ} \mathrm{C}$. Therefore, the pure thermal swelling at 5.0C was obtained by using Eq. (9) and is shown by the dashed line in the top of Figure 4.

Note that the thermal swelling shown in Figure 4 increases due to the raise in temperature, while Li-ion intercalation swelling decreases over the SOC because Li-ion is deintercalated from graphite during discharge.

The core temperature over SOC at 5.0C can then be calculated by using Eq. (8) with the estimated thermal swelling $s_{h}$, the equivalent coefficient of thermal expansion as a function of SOC, the measured surface temperature, and the ambient/reference temperature. The equivalent coefficient of thermal expansion measured at the center of the cell surface (location 3 in Figure 1) was used in the model. We used this calculation because the cell expansion is the largest there and of interest. The core temperature estimated is shown by a dashed-dot line in the middle of Figure 4.

Finally, thermal properties such as the equivalent electric resistivity and the equivalent thermal conductivity for the through-plane direction of the cell were parameterized with the estimated core temperature over SOC. The entropy change of this battery is available [48]. The resistivity, the heat capacity, and the thermal conductivity for the through-plane direction estimated were $1.9 \mathrm{~m} \Omega, 97 \mathrm{JK}^{-1}$, and $0.73 \mathrm{Wm}^{-1} \mathrm{~K}^{-1}$ respectively. The estimate values are reasonable in that the values have similar order of magnitude with values found in the literature. For example, the thermal conductivity for the through-plane direction is $0.61 \mathrm{Wm}^{-1} \mathrm{~K}^{-1}$ in Ref. [49], $0.69 \mathrm{Wm}^{-1} \mathrm{~K}^{-1}$ 
in Ref. [50], and $0.75 \mathrm{Wm}^{-1} \mathrm{~K}^{-1}$ in Ref. [51], which are estimated based on the thickness of each electrode. The heat capacity of battery cells used in HEVs is also $88 \mathrm{JK}^{-1}$ in Ref. [52]. Note that measuring all thermal properties from experiments is very time and cost intensive. The purpose of this study is to create a thermal swelling model, not to identify thermal properties of the cell experimentally.

The core temperature was also estimated using the 1-D heat conduction model in Eq. (3). The measured surface temperature was used to obtain the results shown as diamond symbols in the middle of Figure 4. The model results are in good agreement with the estimated core temperature from Eq. (8). The interesting behavior of the core temperature is between 0.4 and 0.8 SOC. The temperature difference between the core and the surface decreases in this region, even though the Joule heating still exists. To elucidate the reason for this phenomenon, another heat source, namely the entropy change, should be considered as shown in the bottom of Figure 4 [48]. The entropy change is negative in the 0.45 to $0.8 \mathrm{SOC}$ region. This means that the entropy is not a heat source but a heat sink in this region. Moreover, the heat the entropy absorbed is larger than the heat created by the internal resistance. As a result, the temperature difference between the core and the surface decreases in the 0.4 to 0.8 SOC region. In contrast, the core temperature increases drastically in the 0 to 0.2 SOC region due to the synergy of the Joule heat and the entropy heat. Both of them are positive, i.e. exothermic, in this region. In summary, the entropy heat plays a critical role and should be considered in the cell to predict the exact core temperature during operation.

Figure 5 compares measured thermal swelling with estimated thermal swelling by using Eq. (2) during discharge at 5.0C. Two conditions were simulated in the estimation of thermal swelling to elucidate the effect of the temperature distribution through the cell. The first set of results 
assumed that the temperature through the cell (in the $z$ direction) was the same, i.e., the temperature distribution is uniform in the direction perpendicular to the cell surface. Under this assumption, the first term, $\frac{2}{3} \alpha(t) L\left(T_{C}(t)-T_{S}(t)\right)$, of Eq. (2) is zero. The results in this first set are shown in Figure 5 as circles. The second set of results assumed that the temperature distribution though the battery can be obtained using the 1-D heat conduction equation. In this case, Eq. (3) was used to predict the core temperature. The results are shown by triangles in Figure 5. Finally, the third set of results is shown as diamond symbols. They are the estimated thermal swelling at 5.0C by using Eq. (9), which is perhaps the most direct and accurate measurement possible.

A large difference exists between the measured values and the simulation results when the core and the surface temperatures were assumed to be the same. On the contrary, the simulation results which consider the temperature distribution (and hence consider the deference between the surface and the core temperatures) correspond well to the measured values. This demonstrates that the temperature distribution through the cell is not negligible from the thermal swelling perspective (because the equivalent coefficient of thermal expansion is large). Therefore, the temperature distribution through the cell should be accounted for in the thermal swelling model (the first term of Eq. (2)) to accurately predict the thermal swelling.

\subsection{Model validation}

The top of Figure 6 illustrates the total swelling over SOC at a variety of C-rates. The symbols show experimental data, while the solid lines show the sum of the thermal swelling simulated by 
using Eq. (2) and the Li-ion intercalation swelling (i.e., measured swelling at 0.2C) at various Crates. The thermal swelling in the simulation was estimated by using the surface/ambient temperature measured, the core temperature predicted by the 1-D conduction model (Eq. (3)), and the equivalent coefficient of thermal expansion measured at the center of the cell surface (location 3 in Figure 1).

The higher is the C-rate of the battery when discharged, the larger is the residual (thermal) swelling at the end of discharge. This is because the thermal swelling increases as the discharge progress and the temperature increases, while the Li-ion intercalation swelling decreases during discharge as shown in the top of Figure 6. Therefore, large thermal stress is induced not only in the cell but also in the battery pack during high C-rate operation. This thermal stress might be a major reason for the degradation of cycle life during high C-rate operation [53]. Hence, an accurate model to predict the thermal swelling is useful for the efficient control and management of the cells and packs.

The proposed thermal swelling model was shown to accurately predict the thermal swelling at $1.0 \mathrm{C}, 2.0 \mathrm{C}$ and $5.0 \mathrm{C}$ rates, indicating that our approach is reasonable and the estimated thermal properties are accurate. The root-mean-square errors (RMSE) in predicted swelling are $1.13 \mu \mathrm{m}$, $1.23 \mu \mathrm{m}$, and $1.25 \mu \mathrm{m}$ at $1.0 \mathrm{C}, 2.0 \mathrm{C}$, and $5.0 \mathrm{C}$ respectively. The accuracy of the displacement sensor and the thermocouple is $1 \mu \mathrm{m}$ and $0.5^{\circ} \mathrm{C}$ respectively. The accuracy of the thermocouple alone can result in an error over $1 \mu \mathrm{m}$ (base on the measured equivalent coefficient of thermal expansion). Considering the accuracy of sensors, the proposed thermal model accurately predicts the thermal swelling at various C-rates. Moreover, these results justify two hypotheses: (1) the total swelling is a sum of the Li-ion intercalation swelling and the thermal swelling: and (2) the rate dependence of the $\mathrm{Li}$-ion intercalation swelling is negligible. 
For a reliable verification, the total swelling measured is also compared with the total swelling estimated during relaxation period at a variety of C-rates (the bottom of Figure 6). The total swelling estimated is the sum of the thermal swelling calculated by using Eq. (4) and the Li-ion intercalation swelling measured at $0.2 \mathrm{C}$ rate. The solid lines show model predictions, while the symbols represent experimental results. The estimated swelling matches well with the measured swelling at various C-rates. These results show that the proposed model predicts the thermal swelling accurately during the operation and relaxation periods at a variety of C-rates.

The trend of temperature and swelling during the relaxation period at 5.0C are shown to elucidate the dynamic thermal mechanics of the battery cell in Figure 7. The core temperature estimated and the surface temperature measured are shown as a solid line and a dashed line in the top of Figure 7. The initial value of the core temperature, which is essential to solving Eq. (5), was obtained from the simulation result for discharge. The initial value of the core temperature for the relaxation period is the last value of the core temperature estimated using Eq. (3) during the discharge. The initial difference between the core and the surface temperatures is over $1^{\circ} \mathrm{C}$. As the relaxation progresses, the surface and the core temperatures decrease, and the difference between them also decreases to reach the final thermal equilibrium. The surface temperature converges to the ambient/reference temperature. The core temperature converges to the surface temperature. Eventually, both temperatures converge to the ambient/reference temperature after enough relaxation.

The swelling during the relaxation period is shown in the bottom of Figure 7. The dashed line shows the thermal swelling $s_{h}$ estimated by using Eq. (4). The dashed-dot line shows the Li-ion intercalation swelling $s_{L i}$. The dotted line represents the sum of the estimated thermal swelling 
and Li-ion intercalation swelling $\left(s_{h}+s_{L i}\right)$. The solid line is the total swelling measured at 5.0C.

The sum of the thermal swelling and the Li-ion intercalation swelling corresponds well with the total measured swelling. These results also corroborate the two hypotheses, namely: (1) the total swelling is a sum of the Li-ion intercalation swelling and the thermal swelling: and (2) the rate dependence of the Li-ion intercalation swelling is negligible.

The model should be validated at higher C-rate to assess model viability for battery health management in HEVs. The result of the pulse excitation experiment at a high charge rate (39A) was compared to the model prediction (Figure 8 ). The maximum capability (40A) of the battery test equipment was used. The dashed line in the top figure shows the surface temperature measured, whereas the solid line in the top figure represents the core temperature estimated. The dashed line in the bottom figure shows the thermal swelling measured, whereas the solid line in the bottom figure represents the thermal swelling estimated from the model. The entropic heat is negligible in the pulse experiment. The positive and negative current pulse is repeatedly induced to the battery cell and thereby the total amount of heat from the entropy change is zero. Moreover, the SOC is minimally changed during pulse excitation. Therefore, the swelling in this experiment is pure thermal swelling.

The surface temperature measured converged to $29.4^{\circ} \mathrm{C}$ during the pulse excitation because the battery reached the thermally equilibrium state (as seen in the top of Figure 8). The core temperature estimated also converged to $30.7^{\circ} \mathrm{C}$ when the battery reached the thermal equilibrium state. Note that this difference between the core temperature and surface temperature is important to estimate thermally induced swelling considering the large coefficient of thermal expansion on the cell level. This again suggests that the temperature difference through the 
battery cell should be considered to accurately predict the swelling induced from the temperature elevation. The bottom of Figure 8 shows the swelling measured and predicted by the proposed model. The model prediction is in excellent agreement with the measurement. The RMSE between the model and experiment is $1.33 \mu \mathrm{m}$. This experimental validation confirms that the model has high accuracy even at high C-rate. In summary, the model proposed not only accurately predicts the thermal swelling but also is computationally effective.

The proposed model can be used for battery health management or a supervisory controller of electrified vehicles such as HEVs, EVs, and PHEVs. For example, the coupled electrochemicalthermal model predicts the SOC and the surface/core temperature, which are input variables for the proposed thermal swelling model. The proposed model predicts well the amount of thermal swelling due to temperature elevation. Finally, this information can be used for battery health management. This fully coupled model not only increases the operational window but also reduces the overall price of battery cells and packs. However, several temperature sensors should be installed in the hotspot of a battery pack when there are concerns regarding risk due to uncertainties in the prediction of temperature and swelling. In fact, this risk has stimulated the development of an ultrathin sensor array capable of measuring cell expansion and temperature across multiple cells within a battery pack [54]. The state estimates with closed-loop feedback also improve the accuracy of the model prediction [49]. In summary, installing the minimal number of temperature sensors with an accurate model is the best way not only to improve safety and reliability but also to enhance the commercial viability of battery cells and packs considering the stringent safety standards for vehicles. 
Note that this study only considers the dependency of the equivalent coefficient of thermal expansion on the SOC because the swelling at various C-rates was measured at a constant temperature $\left(25^{\circ} \mathrm{C}\right)$. However, the nonlinear characteristics of the equivalent coefficient of thermal expansion at low temperature should be considered if the battery is operated at low temperatures. The Li-ion intercalation swelling also should be modeled to predict total swelling of the cell.

\section{Conclusions}

The thermal characteristics of a battery cell were identified. The observed thermal swelling shows a quadratic behavior in low temperatures, while exhibiting a linear behavior in high temperatures at an unconstrained condition. This nonlinear behavior might be caused by contact surfaces between the electrodes because many gaps can be present among electrode layers. Moreover, the thermal swelling depends on the measured location and the SOC because of the complex shape of the cell components, constraints, and the phase transition of each electrode. Therefore, a variety of perspectives should be considered to accurately predict the thermal swelling of the cell and estimate the equivalent coefficient of thermal expansion because Li-ion battery consists of many materials, and their internal shape and connection inside of the cell is complex.

Based on experimental characterization, a novel thermal swelling model was proposed. The proposed model addresses the dependence of the equivalent coefficient of thermal expansion on the SOC and reflects the temperature distribution through the cell. Simulation results show a 
large difference with the experiment unless considering the temperature distribution through the cell. Through comparison between simulations and experiments, it was verified that the proposed model accurately predicts the thermal swelling at a variety of C-rates during the operation and relaxation periods.

\section{Acknowledgements}

The information, data, or work presented herein was funded in part by the Advanced Research Projects Agency-Energy (ARPA-E), U.S. Department of Energy, under Award Number DEAR0000269.

\section{Disclaimer}

The information, data, or work presented herein was funded in part by an agency of the United States Government. Neither the United States Government nor any agency thereof, nor any of their employees, makes any warranty, express or implied, or assumes any legal liability or responsibility for the accuracy, completeness, or usefulness of any information, apparatus, product, or process disclosed, or represents that its use would not infringe privately owned rights. Reference herein to any specific commercial product, process, or service by trade name, trademark, manufacturer, or otherwise does not necessarily constitute or imply its endorsement, recommendation, or favoring by the United States Government or any agency thereof. The views and opinions of authors expressed herein do not necessarily state or reflect those of the United States Government or any agency thereof. 


\section{References}

[1] M. Bryner, Chemical Engineering Progress. 109 (10) (2013) 36-39.

[2] A. N. Jansen, Chemical Engineering Progress. 109 (10) (2013) 57-64.

[3] B. Scrosati, and J. Garche, Journal of Power Sources. 195 (2010) 2419-2430.

[4] L. B. Lave, and H. L. MacLean, Transportation Research Part D. 7 (2) (2002) 155-162.

[5] F. V. Conte, Elektrotechnik \& Informationstechnik. 123 (10) (2006) 424-431.

[6] T. M. Bandhauer, S. Garimella, and T. F. Fuller, Journal of the Electrochemical Society. 158 (3) (2011) R1-R25.

[7] X. Hu, S. Lin, S. Stanton, and W. Lian, IEEE Transactions on Industry Applications. 47 (4) (2011) 1692-1699.

[8] M.-S. Wu, K. H. Liu, Y.-Y. Wang, and C.-C. Wan, Journal of Power Sources. 109 (2002) 160-166.

[9] S.-C. Chen, Y.-Y. Wang, and C.-C. Wan, Journal of the Electrochemical Society. 153 (4) (2006) A637-A648.

[10] S. Peck, T. Olszanski, S. Zanardelli, and M. Pierce, SAE Int. J. Passeng. Cars - Electron. Electr. Syst. 5 (1) (2012) 154-163.

[11] V. Srinivasan, and C. Y. Wang, Journal of the Electrochemical Society. 150 (1) (2003) A98A106

[12] Y. Chen, and J. W. Evans. Journal of the Electrochemical Society. 143 (9) (1996) 27082712.

[13] S. A. Khateeb, S. Amiruddin, M. Farid, J. R. Selman, S. Al-Hallaj, Journal of Power Sources. 142 (2005) 345-353. 
[14] A. Santucci, A. Sorniotti, and C. Lekakou, Journal of Power Sources. 258 (2014) 395-407.

[15] K. Onda, T. Ohshima, M. Nakayama, K. Fukuda, and T. Araki, Journal of Power Sources. 158 (2006) 535-542.

[16] C. Bindra, V. A. Nalimova, D. E. Sklovsky, W. A. Kamitakahara, and J. E. Fischer, Physical Review B. 57 (9) (1998) 5182-5190.

[17] K. Mukai, Y. Kishida, H. Nozaki, and K. Dohmae, Journal of Power Sources. 224 (2013) 230-235.

[18] S. Komaba, A. Ogata, T. shimizu, and S. Ikemoto, Solid State Ionics. 179 (2008) 1783-1787.

[19] K.-Y. Oh, J. B. Siegel, L. Secondo, S. U. Kim, N. A. Samad, J. Qin, D. Anderson, K. Garikipati, A. Knobloch, B. I. Epureanu, C. W. Monroe, and A. G. Stefanopoulou, Journal of Power Sources. 267 (2014) 197-202.

[20] Y. Koyama, I. Tanaka, H. Adachi, Y. Makimura, and T. Ohzuku, Journal of Power Sources 119-121 (2003) 644-648.

[21] X. Wang, Y. Sone, and S. Kuwajima, Journal of the Electrochemical Society 151 (2) (2004) A273-A280.

[22] J. H. Lee, H. M. Lee, and S. Ahn, Journal of Power Sources 119-121 (2003) 833-837.

[23] X. Wang, Y. Sone, G. Segami, H. Naito, C. Yamada, and K. Kibe, Journal of the Electrochemical Society 154 (1) (2007) A14-A21.

[24] X. Lin, H. E. Perez, J. B. Siegel, A. G. Stefanopoulou, Y. Li, R. D. Anderson, Y. Ding, and M. P. Castanier, IEEE Transactions on Control System Technology. 21 (5) 2013 1745-1755.

[25] X. Lin, H. E. Perez, S. Mohan, J. B. Siegel, A. G. Stefanopoulou, and Y. Ding, and M. P. Castanier, Journal of Power Sources. 257 (2014) 1-11. 
[26] D. Bernardi, E. Pawlikowski, and J. Newman, Journal of the Electrochemical Society. 132 (1) (1985) 5-12.

[27] S. Chen, C. Wan, and Y. Wang, Journal of Power Sources. 140 (2005) 111-24.

[28] Y. Kim, J. B. Siegel, and A. G. Stefanopoulou, 2013 American Control Conference. Washington, USA (2013).

[29] Y. Qi, H. Guo, L. G. Hector, Jr., and A. Timmons, Journal of the Electrochemical Society. 157 (5) (2010) A558-A566.

[30] R. Yazami, and Y. Reynier, Journal of Power Sources. 153 (2006) 312-318.

[31] K. Onda, H. Kameyama, T. Hanamoto, and K. Ito, Journal of the Electrochemical Society. 150 (3) (2003) A285-A291.

[32] N. Zhang, and H. Tang, Journal of Power Sources. 218 (2012) 52-55.

[33] P. Arora and Z. (J.) Zhang. Chem. Rev. 104 (2004) 4419-4462.

[34] D. J. Lacks, and G. C. Rutledge. J. Phys. Chem. 98 (1994) 1222-1231.

[35] K. Nansaka, and Y. Yamauchi, United States Patent US 8216715 (2012).

[36] J. R. Dahn, Physical Review B. 44 (17) (1991) 9170-9177.

[37] T. Ohzuku, Y. Iwakoshi, and K. Sawai, Journal of the Electrochemical Society. 140 (9) (1993) 2490-2498.

[38] J. Sugiyama, T. Tamura, and H. Yamauchi, Journal of Physics: Condensed Matter. 7 (1995) 9755-9764.

[39] V.A. Sethuraman, M.J. Chon, M. Shimshak, N. Van Windle, and P. R. Fuduru, Electrochemistry Communications. 12 (2010) 1614-1617.

[40] Polymer data handbook, Oxford University Press, 1999. 
[41] N. Lagakos, J. Jarzynski, J. H. Cole, and J. A. Bucaro. Journal of Applied Physics. 59 (1986) 4017-4031.

[42] K. Nansaka, and Y. Yamauchi, United States Patent US 8216715 B2 (2012).

[43] S. Mohan, Y. Kim, J. B. Siegel, N. A. Samad, and A. G. Stefanopoulou, Journal of the Electrochemical Society. 161 (14) (2014) A2222-A2231.

[44] B. Sood, M. Osterman, and M. Pecht, 2013 IEEE Symposium on Product Compliance Engineering. Austin, USA (2013).

[45] H. L. Tuller, J. Schoonman, and I. Riess, Kluwer Academic Publishers, 2000.

[46] F. Tietz, Ionics. 5 (1999) 129-139.

[47] R. E. Garcia, Y. M. Chiang, W. C. Carter, P. Limthongkul, and C. M, Bishop, Journal of the Electrochemical Society. 152 (2005) A255-A263.

[48] N. A. Samad, J. B. Siegel, and A. G. Stefanopoulou, Proceedings of the ASME 2014 Dynamic Systems and Control Conference. San Antonio, USA (2014).

[49] Y. Kim, S. Mohan, J. B. Siegel, A. G. Stefanopoulou, and Y. Ding, IEEE transactions on control systems technology 22 (6) (2014) 2277- 2286.

[50] H. Khasawneh, J. Neal, M. Canova, Y. Guezennec, R. Wayne, J. Taylor, M. Smalc, and J. Norley, IMECE2011 Denver, USA (2011) 421-428.

[51] S.C. Chen, C.C. Wan, and Y. Y. Wang, JPS 140 (1) (2005) 111-124.

[52] R. Beger, H. Wenzl, H.P. Beck, M. Jiang, D. Ohms, G. Schaedlich, EVS24, Stavanger, Norway (2009) 342-351.

[53] G. Ning, B. Haran, and B. N. Popov, Journal of Power Sources. 117 (2003) 160-169. 
[54] A. Knobloch, J. Siegel, A. Stefanopoulou, C. Monroe, B. Epureanu, K. Garipkipati, and D. Anderson, Proceeding of the Advanced Automotive Batteries Conference 2014. Atlanta, USA (2014). 


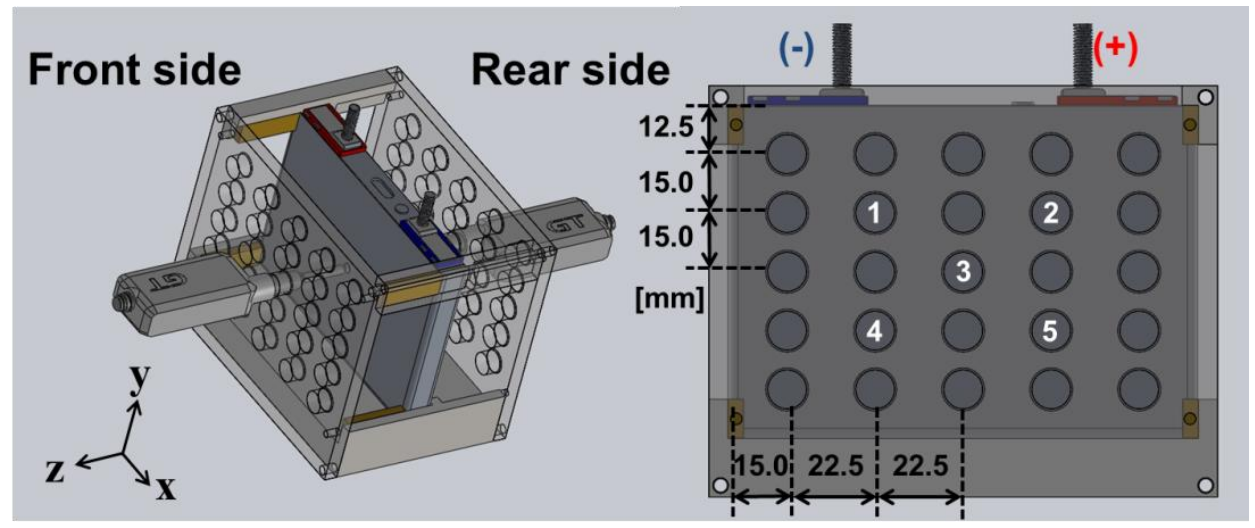

Figure 1. (Color online) Schematic diagram of the experimental setup showing the fixture, the cell, and the sensor locations 1-5. 


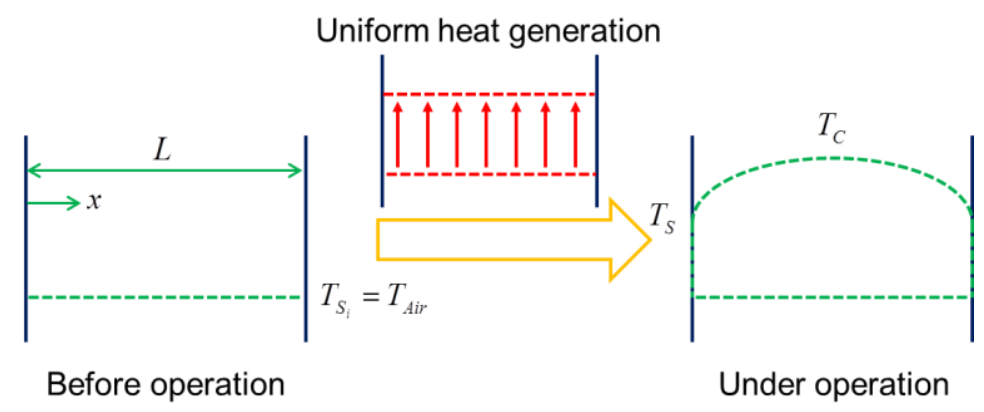

Figure 2. (Color online) Temperature distribution through the cell before and under operation. 
(a)

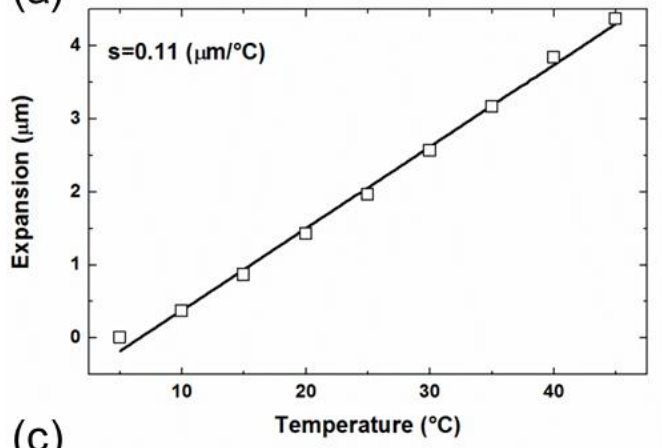

(c)

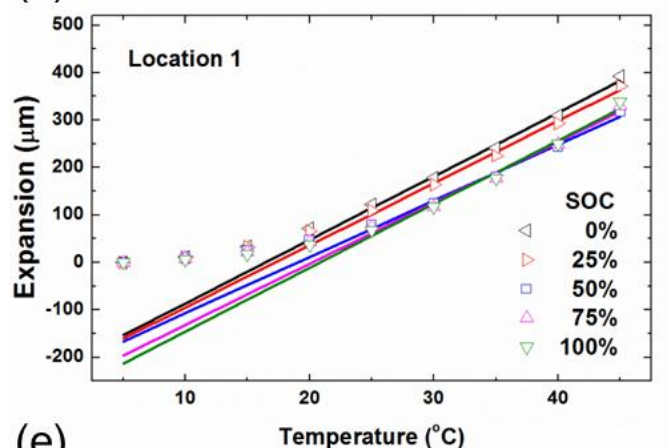

(e)

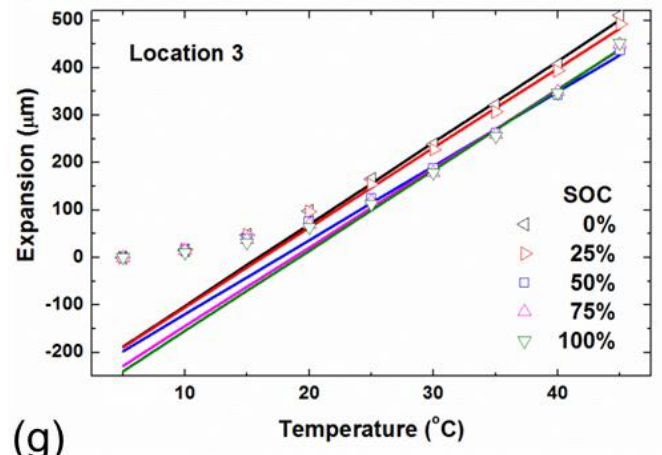

(g)

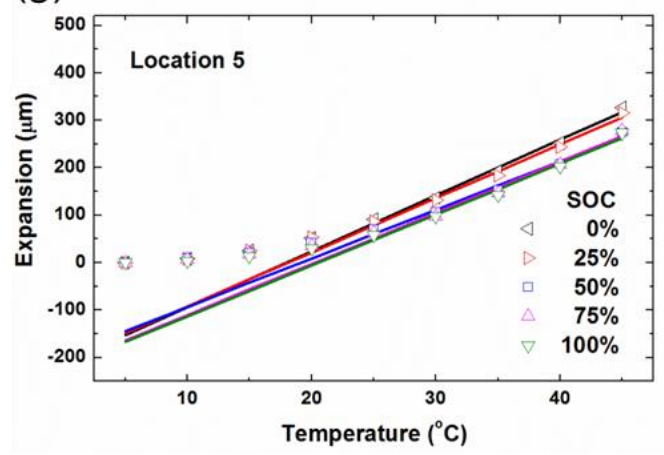

(b)

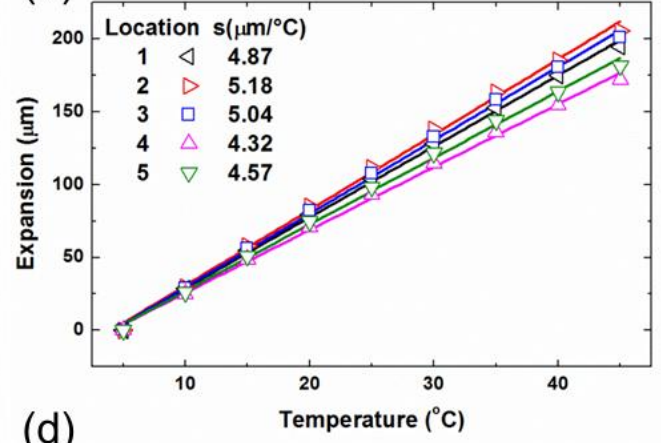

(d)

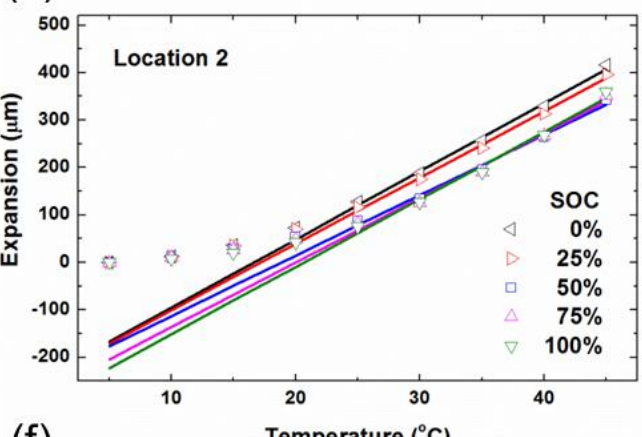

(f)

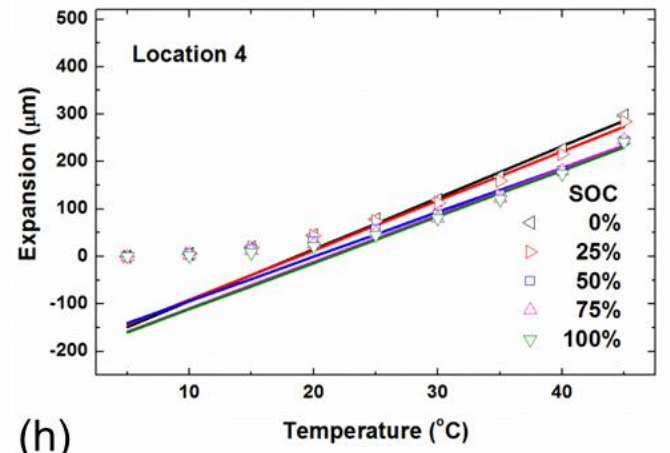

(h)

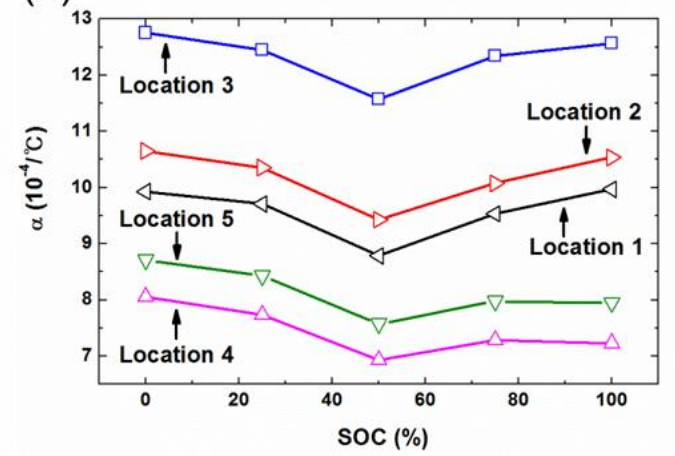

Figure 3. (Color online) Thermal expansion of (a) the sensor, (b) the fixture, (c) - (g) the cell at location $1-5$, and (h) the equivalent coefficient of thermal expansion of the cell as a function of SOC at a variety of locations; the symbols and the solid lines represent measured values and least-square fitted lines from the measured values in (a) and (b); the symbols and the solid lines indicate calibrated thermal swelling of the cell and least-square fitted lines from measured data ranging from $25^{\circ} \mathrm{C}$ to $45^{\circ} \mathrm{C}$ in (c)-(g). 


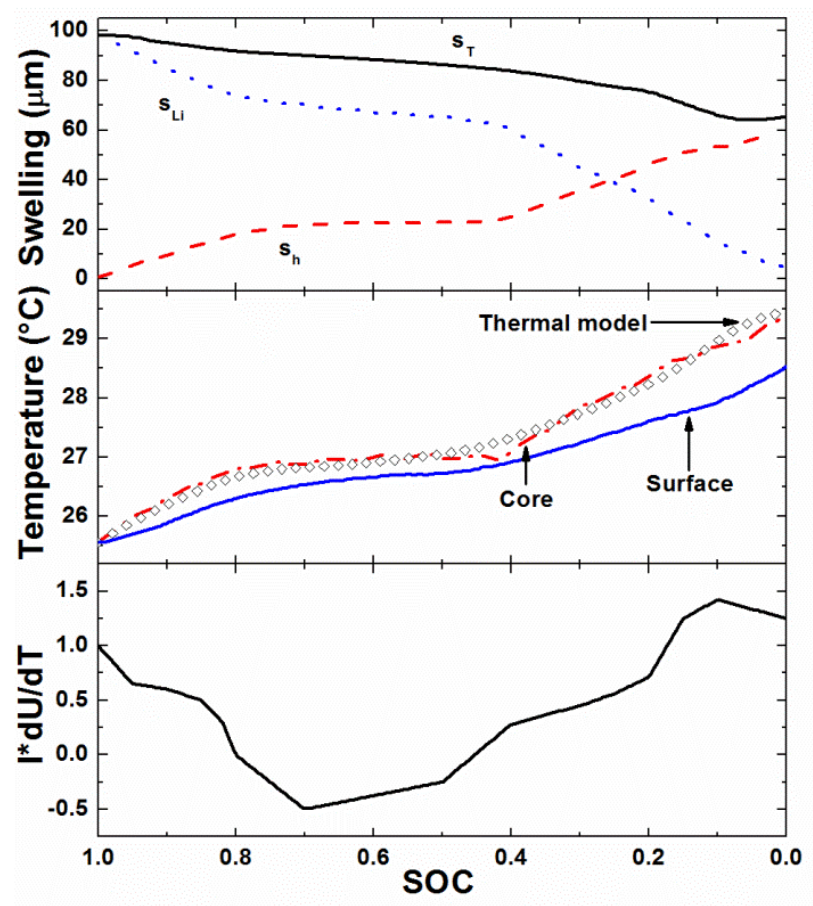

Figure 4. (Color online) (Top) Swelling, (middle) temperature, and (bottom) entropy change versus SOC at the center of the cell during a 5.0C discharge; the solid line, the dotted line, and the dashed line represent the total swelling, the Li-ion intercalation swelling, and the thermal swelling in the top of the figure; the solid line, the dashed-dot line, and the diamond symbol indicate the surface temperature measured, the core temperature calculated by using Eq. (8), and the core temperature estimated by the 1-D heat conduction model (Eq. (3)) in the middle of the figure. 


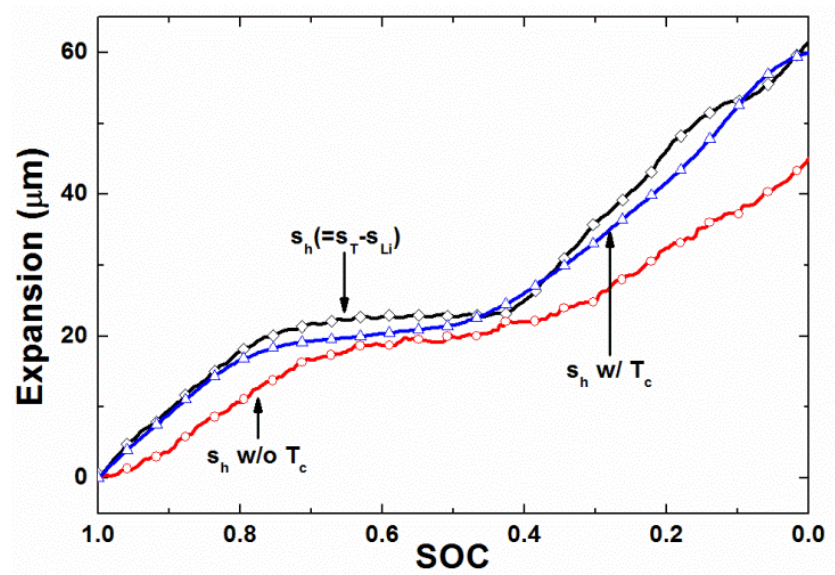

Figure 5. (Color online) Comparison between experimental and simulated thermal swelling $s_{h}$ during discharge at 5.0C; the diamond, the triangle, and the circle symbols represent the calculated thermal swelling from experiment by using Eq. (9), the thermal swelling estimated assuming the nonuniform temperature distribution through the battery cell, and the thermal swelling estimated assuming the uniform temperature distribution through the battery cell. 

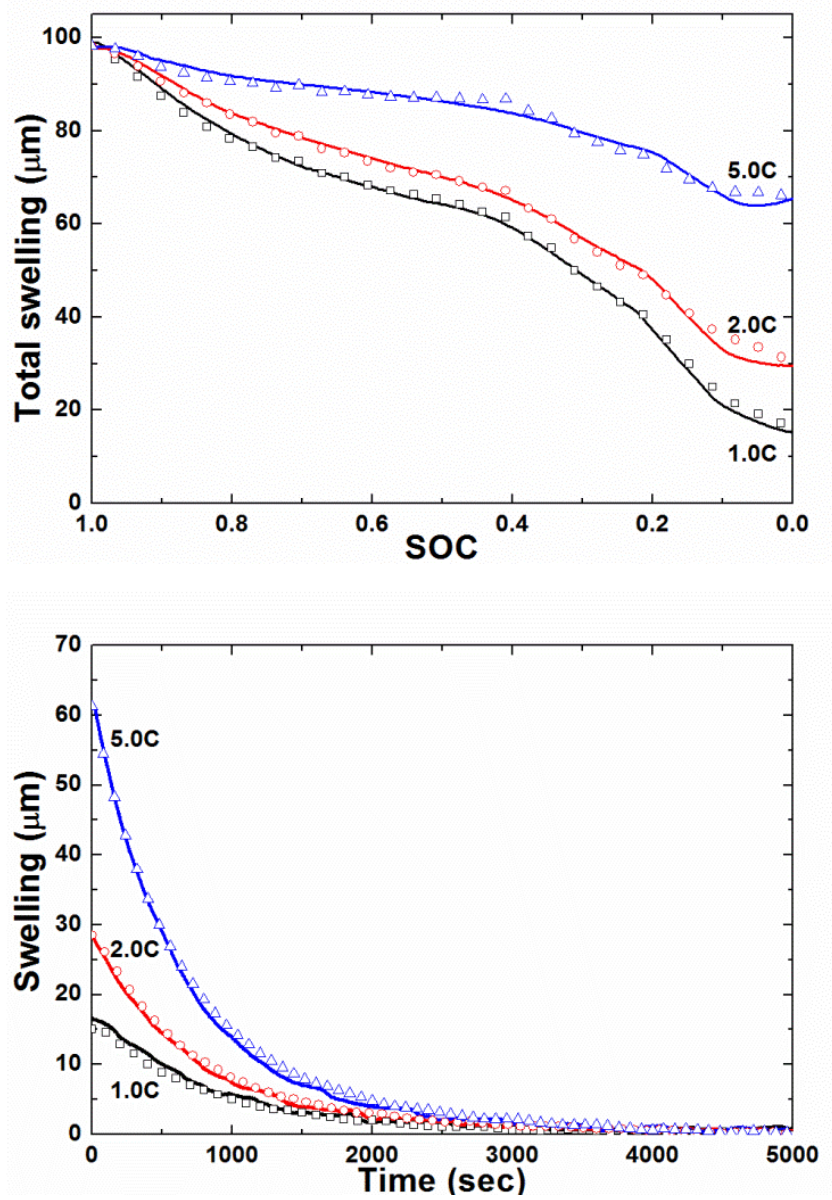

Figure 6. (Color online) (Top) Total swelling versus SOC at a variety of C-rates during operation and (bottom) total swelling right after discharges at a variety of C-rates during the open-circuit relaxation period on the center of the surface of the cell (location 3 in Figure 1); the symbols show experimental results, whereas the solid lines show model predictions. 


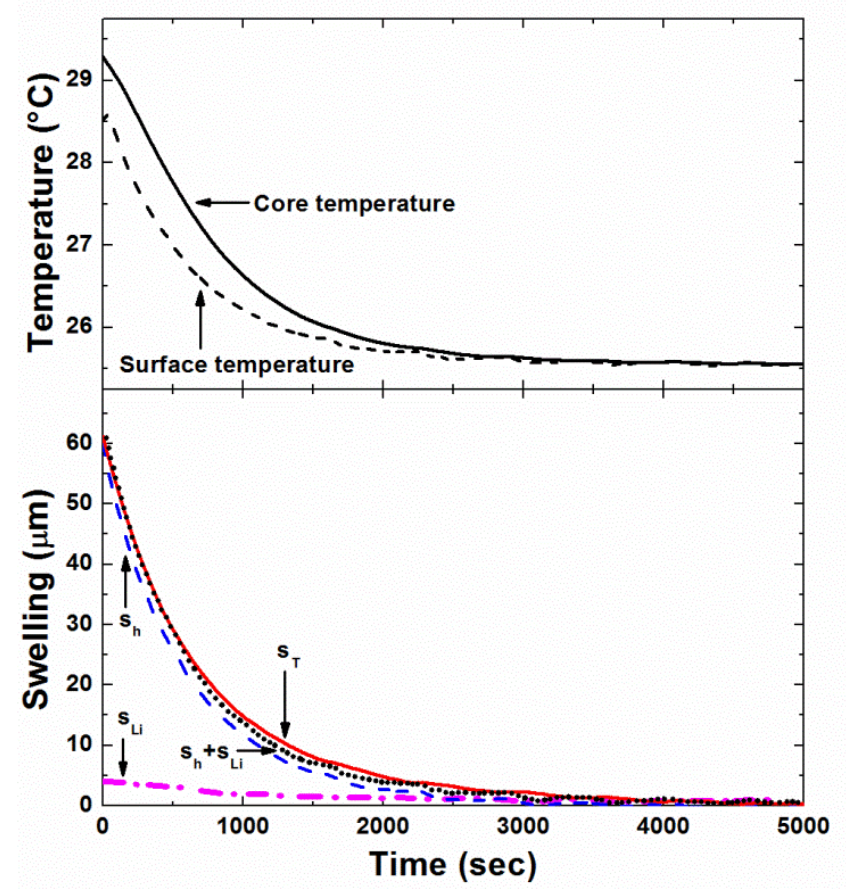

Figure 7. (Color online) (Top) Temperature and (bottom) swelling over time right after a 5.0C discharge during the open-circuit rest period on the center of the surface of the battery (location 3 in Figure 1); the solid line and the dashed line denote the core temperature estimated and the surface temperature measured in the top of figure; the solid line, the dashed line, the dashed-dot line, and the dotted line represent the total swelling measured, the thermal swelling $s_{h}$ estimated by using Eq. (4), the Li-ion intercalation swelling $s_{L i}$, and the sum of the estimated thermal swelling and Li-ion intercalation swelling $\left(s_{h}+s_{L i}\right)$ in the bottom of figure. 


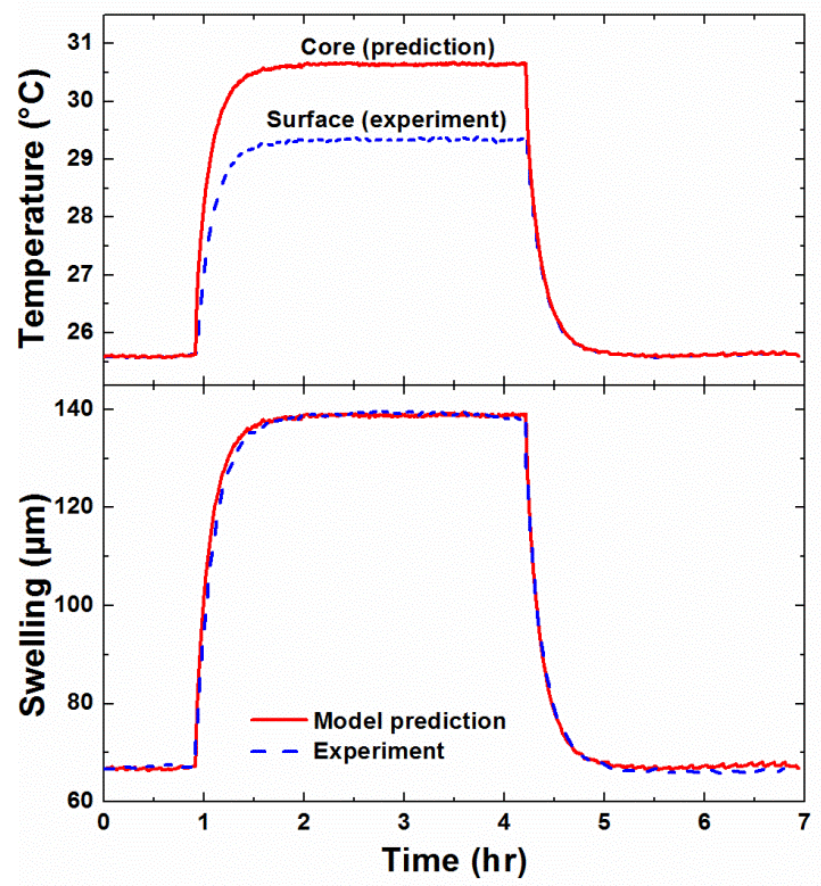

Figure 8. (Color online) (Top) Temperature and (bottom) swelling over time for 7.8C (39A) pulse excitation experiment; swelling is measured at the center of the surface of the battery (location 3 in Figure 1); the dashed line in the top figure shows the surface temperature measured, whereas the solid line in the top figure represents the core temperature estimated; the dashed line in the bottom figure shows the thermal swelling measured, whereas the solid line in the bottom figure represents the thermal swelling estimated from the model. 


\section{Figure captions}

Figure 1. (Color online) Schematic diagram of the experimental setup showing the fixture, the cell, and the sensor locations 1-5.

Figure 2. (Color online) Temperature distribution through the cell before and under operation.

Figure 3. (Color online) Thermal expansion of (a) the sensor, (b) the fixture, (c) - (g) the cell at location $1-5$, and the equivalent coefficient of thermal expansion $\alpha$ of the cell as a function of SOC at a variety of locations (h).

Figure 4. (Color online) (Top) Swelling, (middle) temperature, and (bottom) entropy change versus SOC at the center of the cell during a 5.0C discharge; the solid line, the dotted line, and the dashed line represent the total swelling, the Li-ion intercalation swelling, and the thermal swelling in the top of the figure; the solid line, the dashed-dot line, and the diamond symbol indicate the surface temperature measured, the core temperature calculated by using Eq. (8), and the core temperature estimated by the 1-D heat conduction model (Eq. (3)) in the middle of the figure.

Figure 5. (Color online) Comparison between experimental and simulated thermal swelling $s_{h}$ during discharge at 5.0C; the diamond, the triangle, and the circle symbols represent the calculated thermal swelling from experiment by using Eq. (9), the thermal swelling estimated assuming the nonuniform temperature distribution through the battery cell, and the thermal swelling estimated assuming the uniform temperature distribution through the battery cell.

Figure 6. (Color online) (Top) Total swelling versus SOC at a variety of C-rates during operation and (bottom) total swelling right after discharges at a variety of C-rates during the open-circuit relaxation period on the center of the surface of the cell (location 3 in Figure 1); the symbols show experimental results, whereas the solid lines show model predictions.

Figure 7. (Color online) (Top) Temperature and (bottom) swelling over time right after a 5.0C discharge during the open-circuit rest period on the center of the surface of the battery (location 3 in Figure 1); the solid line and the dashed line denote the core temperature estimated and the surface temperature measured in the top of figure; the solid line, the dashed line, the dashed-dot line, and the dotted line represent the total swelling measured, the thermal swelling $s_{h}$ estimated by using Eq. (4), the Li-ion intercalation 
swelling $s_{L i}$, and the sum of the estimated thermal swelling and Li-ion intercalation swelling $\left(s_{h}+s_{L i}\right)$ in the bottom of figure.

Figure 8. (Color online) (Top) Temperature and (bottom) swelling over time for 7.8C (39A) pulse excitation experiment; swelling is measured at the center of the surface of the battery (location 3 in Figure 1); 